\title{
EEG markers for early detection and characterization of vascular dementia during working memory tasks
}

\begin{abstract}
The aim of the this study was to reveal markers using spectral entropy (SpecEn), sample entropy (SampEn) and Hurst Exponent $(\mathrm{H})$ from the electroencephalography (EEG) background activity of 5 vascular dementia ( $\mathrm{VaD}$ ) patients, 15 stroke-related patients with mild cognitive impairment (MCI) and 15 control healthy subjects during a working memory (WM) task. EEG artifacts were removed using independent component analysis technique and wavelet technique. With ANOVA $(\mathrm{p}<0.05)$, SpecEn was used to test the hypothesis of slowing the EEG signal down in both $\mathrm{VaD}$ and MCI compared to control subjects, whereas the SampEn and $\mathrm{H}$ features were used to test the hypothesis that the irregularity and complexity in both $\mathrm{VaD}$ and MCI were reduced in comparison with control subjects. SampEn and $\mathrm{H}$ results in reducing the complexity in $\mathrm{VaD}$ and $\mathrm{MCI}$ patients. Therefore, SampEn could be the EEG marker that associated with $\mathrm{VaD}$ detection whereas $\mathrm{H}$ could be the marker for stroke-related MCI identification. EEG could be as a valuable marker for inspecting the background activity in the identification of patients with $\mathrm{VaD}$ and stroke-related MCI.
\end{abstract}

Keyword: Electroencephalography; Hurst exponent; ICA-WT; mild cognitive impairment; Sample entropy; Spectral entropy; Vascular dementia 